\title{
Placement of a double-lumen tube using LMA C Trach and an exchanger catheter in difficult airway intubation -A case report-
}

\author{
Lale Karabiyik \\ Department of Anesthesiology and Intensive Care, Gazi University School of Medicine, Ankara, Turkey
}

During insertion of the double lumen tube in patients with cervical vertebral fixation, the cervical neutral position should be maintained. Although flexible fiberoptic bronchoscopic intubation is the gold standard, novel techniques are needed to facilitate intubation of patients with cervical vertebral fixation in neutral position according to institutional capabilities. In this case report, insertion of the double lumen tube in the neutral position using LMA CTrach and an airway exchanger catheter in a thoracotomy patient with extremely limited head and neck motion due to fixation of the cervical vertebrae is presented. (Korean J Anesthesiol 2012; 62: 565-567)

Key Words: Airway management, Difficult airway, Equipment, Laryngeal masks, Lung separation.

Many thoracic surgeries necessitate lung isolation and one lung ventilation $(\mathrm{OLV})$ to enhance the surgical view. One-lung ventilation in the thoracic surgical patient can be achieved with the use of a double-lumen endotracheal tube (DLT) or an independent bronchial blocker. In practice, due to their double-curved shapes and wide external diameters, intubation with double lumen tubes is normally more difficult than with single lumen tubes. Patients with difficult intubation further complicate endobronchial intubation with DLT, an already challenging process for the anesthesiologists [1-3].

Patients with cervical vertebra fractures due to trauma are inserted with metal plates to prevent spinal cord injuries. Intubation is complicated by limited neck motion due to the fixation provided by these plates. Patients in such conditions should be intubated while maintaining the head and neck in a neutral position.

Identification of the potentially difficult airway during the preoperative evaluation allows the planning and selection of the appropriate lung isolation device. Flexible fiberoptic intubation, airway scope, trachlight, bronchial stylet, lighted stylet, blockers and proseal laryngeal mask were previously utilized with success in cases involving difficult intubation with DLT [4-7].

In this case report, placement of the DLT in a neutral position in a thoracotomy patient with limited neck motion due to cervical vertebral fixation is presented.

Received: April 25, 2011. Revised: June 16, 2011. Accepted: June 20, 2011.

Corresponding author: Lale Karabiyik, M.D., Ph.D., Department of Anesthesiology and Intensive Care, Gazi University School of Medicine, Ankara 06500, Turkey. Tel: 90-312-2025348, Fax: 90-312-2024166, E-mail: lalekarabiyik@yahoo.com

(C) This is an open-access article distributed under the terms of the Creative Commons Attribution Non-Commercial License (http:// creativecommons.org/licenses/by-nc/3.0/), which permits unrestricted non-commercial use, distribution, and reproduction in any medium, provided the original work is properly cited. 


\section{Case Report}

A 65 year old male patient $(174.5 \mathrm{~cm}$ in height, $77 \mathrm{~kg}$ in weight) with American Society of Anesthesiologists physical status classification II was scheduled for left lung upper lobe wedge resection under the left OLV with a DLT. Mouth opening was $3.5 \mathrm{~cm}$ and the Mallampati score was assessed as class 2 in the patient with normal teeth status. However, the head and neck motion, and especially extension were found to be highly limited $\left(<30^{\circ}\right)$ by the bilateral metal plates and screws placed on cervical spine from vertebrae 2 to 6 following a trauma sustained 16 years ago (Fig. 1). There were neither neurological sequel or complications after the cervical spine fracture and fixation operation. Also, there were no signs of neurological symptoms during neck movement. Therefore, intubation was planned to be performed without laryngoscopy to maintain the head and neck in the neutral position.

Anesthesia was induced by $1 \mathrm{mg} / \mathrm{kg}$ of propofol and 0.6 $\mathrm{mg} / \mathrm{kg}$ of rocuronium intravenously, and maintained by isoflurane in pure oxygen. Airway was maintained with face mask ventilation after muscle relaxation. Thereafter, ventilation was achieved through an anesthesia circuit by inserting a LMA Ctrach ${ }^{\mathrm{TM}}$ (LMA North America Inc, San Diego, USA) laryngeal mask number 4 after applying lubricant on the back side. Following the deconnection of the laryngeal mask from the anesthesia circuit upon achieving rima glottis view on the screen, a lubricated spiral tube number 7 was viewed on the screen and inserted into the trachea on the first attempt. LMA CTrach ${ }^{\mathrm{TM}}$ was then removed with the aid of a stabilizing rod. After the patient was ventilated for a few minutes, a 14 Fr/83 cm tube exchanger catheter (Cook Airway Exchange Catheter, Cook Incorporated, With Rapi-Fit ${ }^{\circledR}$ Adapters, Cook, USA) was insterted inside the tube. The single lumen tube was withdrawn and a lubricated left DLT number 37 (Broncho-Cath, Mallinckrodt Medical Athlone, Ireland) was advanced into the trachea through the tube exchanger catheter. After realizing
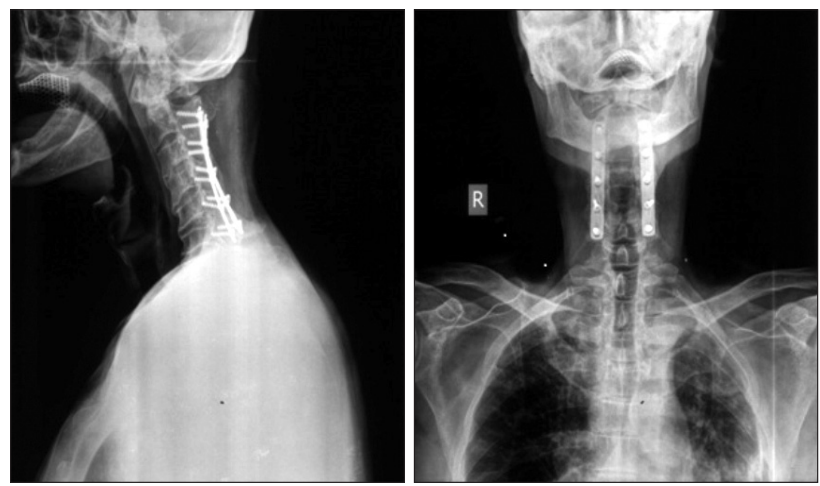

Fig. 1. Lateral and anteroposterior cervical x-ray of the patient. on the first attempt that it was on the right side, the tube was withdrawn up to the carina and successfully inserted into the left main bronchus on the second attempt. After the tube position was confirmed by auscultation, the patient was moved to a lateral decubitus position. During this procedure, oxygen saturation was maintained at more than $95 \%$. Thoracotomy was performed and OLV was successfully applied on the patient.

\section{Discussion}

Special algorithms for lung separation have been defined in such patients, because in difficult intubation, DLT is expected to be even more difficult [1]. The flexible fiberoptic bronchoscope was not available in our clinic during the preparation of this case and therefore, taking into consideration the equipment available, intubation with a single lumen tube through LMA CTrach followed by substitution with a DLT through a tube exchanger catheter was planned and successfully executed.

LMA-Fastrach was designed as a laryngeal mask to facilitate tracheal intubation. LMA CTrach ${ }^{\mathrm{TM}}$, on the other hand, is the modified version of the LMA Fastrach ${ }^{\mathrm{TM}}$ with two fiberoptic objects added to the tip of the airway tube. It has a screen that provides continuous view during tracheal intubation. Intubation can be achieved with a single lumen tube in a neutral position without a laryngoscope through both LMA-Fastrach and LMA $\mathrm{CTrach}^{\mathrm{TM}}$. However, insertion of the DLT with a specific shape into the trachea is not possible through LMAs providing such intubation.

In the event that intubation is achieved with a single lumen tube, it is recommended that either an endobroncheal blocker is inserted through this tube or exchanged with a DLT [1]. In our case, we did not prefer the former approach because had an endobroncheal blocker been applied, its location would have to be confirmed with a flexible fiberoptic bronchoscope. The exchange catheter proposed in the other option needs to be of a size and length compatible with the tube to be exchanged. In our case, we preferred the latter method due to availability of an exchange cathether of a size and length suitable for passing through the endobrochial lumen of the DLT considered for exchange.

Algorithms recommended for intubation with DLT with difficult intubations include tube exchange through exchange catheter practices [1]. However, it is recommended that a catheter no shorter than $70 \mathrm{~cm}$ and with atraumatic properties (i.e. softtipped) be utilized for this purpose. Iatrogenic tracheobronchial ruptures due to intubation with an endobronchial doublelumen tubes are rare but can be life threatening [8,9]. Also, tracheal laceration after the use of an airway exchange catheter for DLT placement has been reported previously [10]. In our case, the catheter used for tube exchange was of sufficient 
length and soft-tipped; no complications were observed after these procedures.

Although flexible fiberoptic intubation is accepted as the gold standard, intubation in our case was achieved through a CTrach ${ }^{\mathrm{TM}}$ LMA without laryngoscopy due to temporary unavailability of the flexible bronchoscope in our institution. The standard single lumen tube was then exchanged for a DLT by using a tube exchanger catheter. Neutral position of the fixed cervical vertebrae was maintained throughout these attempts.

In conclusion, when a small-caliber fiberoptic bronchoscope is unavailable, solutions to overcome the difficulty of intubation with DLT should be devised within the frame of the practitioner experience and resources of each institution.

\section{References}

1. Brodsky JB. Lung separation and the difficult airway. Br J Anaesth 2009; 103 Suppl 1: i66-75.

2. Compos JH. Lung isolation techniques for patients with difficult airway. Curr Opin Anaesthesiol 2010; 23: 12-7.

3. Cohen E. Methods of lung separation. Minerva Anesthesiol 2004; 70:313-8.
4. Orhan ME, Gözübüyük A, Sizlan A, Dere U. Unexpected difficult intubation due to lingual tonsillar hyperplasia in a thoracotomy patient: intubation with the double-lumen tube using stylet and fiberoptic bronchoscopy. J Clin Anesth 2009; 21: 439-41.

5. Poon KH, Liu EH. The Airway Scope for difficult double-lumen tube intubation. J Clin Anesth 2008; 20: 319.

6. Chen KY, Tsao SL, Lin SK, Wu HS. Double-lumen endobronchial tube intubation in patients with difficult airways using Trachlight and a modified technique. Anesth Analg 2007; 105: 1425-6.

7. O'Connor CJ, O'Connor TA. Use of lighted stylets to facilitate insertion of double-lumen endobronchial tubes in patients with difficult airway anatomy. J Clin Anesth 2006; 18: 616-9.

8. Thomas V, Neustein SM. Tracheal laceration after the use of an airway exchange catheter for double-lumen tube placement. J Cardiothorac Vasc Anesth 2007; 21: 718-9.

9. Kim J, Lim T, Bahk JH. Tracheal laceration during intubation of a double-lumen tube and intraoperative fiberoptic bronchoscopic evaluation through an LMA in the lateral position -A case reportKorean J Anesthesiol 2011; 60: 285-9.

10. Kim HK, Jun JH, Lee HS, Choi YR, Chung MH. Left mainstem bronchial rupture during one-lung ventilation with Robertshaw double lumen endobronchial tube -A case report-. Korean J Anesthesiol 2010; 59 Suppl: S21-5. 